\title{
Topological susceptibility in (2+1)-flavor lattice QCD with overlap fermion
}

\author{
T.W. Chiu ${ }^{* a} \nmid$ S. Aoki ${ }^{b, c}$, S. Hashimoto ${ }^{e, f}$, T.H. Hsieh ${ }^{g}$, T. Kaneko ${ }^{e, f}$, H. Matsufuru ${ }^{e}$, \\ J. Noaki ${ }^{e}$, T. Onogi ${ }^{h}$, N. Yamada ${ }^{e, f}$ (for JLQCD and TWQCD Collaborations) \\ ${ }^{a}$ Physics Department, Center for Quantum Science and Engineering, and Center for Theoretical \\ Sciences, National Taiwan University, Taipei 10617, Taiwan \\ ${ }^{b}$ Graduate School of Pure and Applied Sciences, University of Tsukuba, Tsukuba 305-8571, \\ Japan \\ ${ }^{c}$ Riken BNL Research Center, Brookhaven National Laboratory, Upton, NY11973, USA \\ ${ }^{e}$ High Energy Accelerator Research Organization (KEK), Tsukuba 305-0801, Japan \\ ${ }^{f}$ School of High Energy Accelerator Science, The Graduate University for Advanced Studies \\ (Sokendai), Tsukuba 305-0801, Japan \\ ${ }^{g}$ Research Center for Applied Sciences, Academia Sinica, Taipei 115, Taiwan \\ ${ }^{h}$ Yukawa Institute for Theoretical Physics, Kyoto University, Kyoto 606-8502, Japan
}

\begin{abstract}
We determine the topological susceptibility $\chi_{t}$ in the topologically-trivial sector generated by lattice simulations of $N_{f}=2+1$ QCD with overlap Dirac fermion, on a $16^{3} \times 48$ lattice with lattice spacing $\sim 0.11 \mathrm{fm}$, for five sea quark masses $m_{q}$ ranging from $m_{s} / 6$ to $m_{s}$ (where $m_{s}$ is the physical strange quark mass). The $\chi_{t}$ is extracted from the plateau (at large time separation) of the 2-point and 4-point time-correlation functions of the flavor-singlet pseudoscalar meson $\eta^{\prime}$, which arises from the finite size effect due to fixed topology. In the small $m_{q}$ regime, our result of $\chi_{t}$ agrees with the chiral effective theory. Using the formula $\chi_{t}=\Sigma\left(m_{u}^{-1}+m_{d}^{-1}+m_{s}^{-1}\right)^{-1}$ by Leutwyler-Smilga, we obtain the chiral condensate $\Sigma^{\overline{\mathrm{MS}}}(2 \mathrm{GeV})=[253(4)(6) \mathrm{MeV}]^{3}$.
\end{abstract}

The XXVI International Symposium on Lattice Field Theory

July 14-192008

Williamsburg, Virginia, USA

\footnotetext{
* Speaker.

†Email: twchiu@phys.ntu.edu.tw
} 


\section{Introduction}

In Quantum Chromodynamics (QCD), the topological susceptibility $\left(\chi_{t}\right)$ is the most crucial quantity to measure the topological charge fluctuations of the QCD vacuum, which plays an important role in breaking the $U_{A}(1)$ symmetry. Theoretically, $\chi_{t}$ is defined as

$$
\chi_{t}=\int d^{4} x\langle\rho(x) \rho(0)\rangle, \quad \rho(x)=\frac{1}{32 \pi^{2}} \varepsilon_{\mu v \lambda \sigma} \operatorname{tr}\left[F_{\mu v}(x) F_{\lambda \sigma}(x)\right]
$$

where $\rho(x)$ is the topological charge density expressed in term of the matrix-valued field tensor $F_{\mu \nu}$. With mild assumptions, Witten [1] and Veneziano [2] obtained a relationship between the topological susceptibility in the quenched approximation and the mass of $\eta^{\prime}$ meson (flavor singlet) in full QCD with $N_{f}$ degenerate flavors, namely, $\chi_{t}$ (quenched) $=f_{\pi}^{2} m_{\eta^{\prime}}^{2} /\left(4 N_{f}\right)$ where $f_{\pi}=131$ $\mathrm{MeV}$, the decay constant of pion. This implies that the mass of $\eta^{\prime}$ is essentially due to the axial anomaly relating to non-trivial topological charge fluctuations, which can turn out to be nonzero even in the chiral limit, unlike those of the (non-singlet) approximate Goldstone bosons.

Using the Chiral Perturbation Theory (ChPT), Leutwyler and Smilga [3] obtained the following relation in the chiral limit

$$
\chi_{t}=\frac{\Sigma}{\left(\frac{1}{m_{u}}+\frac{1}{m_{d}}+\frac{1}{m_{s}}\right)}+\mathscr{O}\left(m_{u}^{2}\right), \quad\left(N_{f}=2+1\right),
$$

where $m_{u}, m_{d}$, and $m_{s}$ are the quark masses, and $\Sigma$ is the chiral condensate. This implies that in the chiral limit $\left(m_{u} \rightarrow 0\right)$ the topological susceptibility is suppressed due to internal quark loops. Most importantly, (1.2) provides a viable way to extract $\Sigma$ from $\chi_{t}$ in the chiral limit.

From (1.1), one obtains

$$
\chi_{t}=\frac{\left\langle Q_{t}^{2}\right\rangle}{\Omega}, \quad Q_{t} \equiv \int d^{4} x \rho(x)
$$

where $\Omega$ is the volume of the system, and $Q_{t}$ is the topological charge. Thus, one can obtain $\chi_{t}$ by counting the number of gauge configurations for each topological sector. Obviously, for a set of gauge configurations with $Q_{t}=0$, it gives $\chi_{t}=0$. However, even for a topologically-trivial gauge configuration, it may possess non-trivial topological excitations in sub-volumes. Thus, one can measure $\chi_{t}$ using the correlation of the topological charges of two sub-volumes.

In general, for any topological sector with $Q_{t}$, using saddle point expansion on the QCD partition function in a finite volume, it can be shown that [4]

$$
\lim _{|x| \rightarrow \infty}\langle\rho(x) \rho(0)\rangle=\frac{1}{\Omega}\left(\frac{Q_{t}^{2}}{\Omega}-\chi_{t}-\frac{c_{4}}{2 \chi_{t} \Omega}\right)+\mathscr{O}\left(\Omega^{-3}\right),
$$

where $c_{4}=-\frac{1}{\Omega}\left[\left\langle Q_{t}^{4}\right\rangle_{\theta=0}-3\left\langle Q_{t}^{2}\right\rangle_{\theta=0}^{2}\right]$. However, for lattice QCD, it is difficult to extract $\rho(x)$ and $Q_{t}$ unambiguously from the gauge link variables, due to their rather strong fluctuations.

To circumvent this difficulty, one may consider the Atiyah-Singer index theorem [5]

$$
Q_{t}=n_{+}-n_{-}=\operatorname{index}(\mathscr{D})
$$

where $n_{ \pm}$is the number of zero modes of the massless Dirac operator $\mathscr{D} \equiv \gamma_{\mu}\left(\partial_{\mu}+i g A_{\mu}\right)$ with \pm chirality. Since $\mathscr{D}$ is anti-Hermitian and chirally symmetric, its nonzero eigenmodes must come 
in complex conjugate pairs (i.e., $\mathscr{D} \phi=i \lambda \phi$ implies $\mathscr{D} \gamma_{5} \phi=-i \lambda \gamma_{5} \phi$, for $\lambda=\lambda^{*} \neq 0$ ) with zero chirality $\left(\int d^{4} x \phi^{\dagger} \gamma_{5} \phi=0\right)$. Thus one can obtain the identity

$$
n_{+}-n_{-}=\int d^{4} x m \operatorname{tr}\left[\gamma_{5}(\mathscr{D}+m)^{-1}(x, x)\right],
$$

by spectral decomposition, where the nonzero modes drop out due to zero chirality. In view of (1.4) and (1.5), one can regard $m \operatorname{tr}\left[\gamma_{5}(\mathscr{D}+m)^{-1}(x, x)\right]$ as topological charge density, to replace $\rho(x)$ in the measurement of $\chi_{t}$.

For lattice QCD, it is well-known that the overlap Dirac operator [6, 7] in a topologically non-trivial gauge background possesses exact zero modes (with definite chirality) satisfying the Atiyah-Singer index theorem. Writing the massive overlap Dirac operator as

$$
D(m)=\left(m_{0}+\frac{m}{2}\right)+\left(m_{0}-\frac{m}{2}\right) \gamma_{5} \frac{H_{w}\left(-m_{0}\right)}{\sqrt{H_{w}^{2}\left(-m_{0}\right)}},
$$

where $H_{w}\left(-m_{0}\right)$ is the standard Hermitian Wilson operator with negative mass $-m_{0}\left(0<m_{0}<2\right)$, then the topological charge density can be defined as

$$
\rho_{m}(x)=m \operatorname{tr}\left[\gamma_{5}\left(D_{c}+m\right)_{x, x}^{-1}\right],
$$

where $\left(D_{c}+m\right)^{-1}$ is the valence quark propagator with quark mass $m$, and $D_{c}$ is a chirally symmetric operator relating to $D(0)$ by $D_{c}=D(0)\left[1-D(0) /\left(2 m_{0}\right)\right]^{-1}$ [8]. Here $\rho_{m}(x)$ is justified to be topological charge density, since it can be shown that $\sum_{x} \rho_{m}(x)=n_{+}-n_{-}$, which is similar to its counterpart in continuum, (1.5).

Now we can replace $\rho(x)$ with $\rho_{1}(x)$, and use (1.3) to extract $\chi_{t}$ for any topological sector. However, on a finite lattice, it is contaminated by $m_{\pi}, m_{\eta^{\prime}}$ and any states which can couple to $\left\langle\rho_{1}(x) \rho_{1}(0)\right\rangle$. An alternative is to consider the correlator of the flavor-singlet pseudoscalar meson $\eta^{\prime}[4]$

$$
\lim _{\left|x_{1}-x_{2}\right| \gg 1} m_{q}^{2}\left\langle\eta^{\prime}\left(x_{1}\right) \eta^{\prime}\left(x_{2}\right)\right\rangle_{Q}=-\frac{\chi_{t}}{\Omega}\left(1-\frac{Q^{2}}{\chi_{t} \Omega}+\frac{c_{4}}{2 \chi_{t}^{2} \Omega}\right)+\mathscr{O}\left(e^{-m_{\eta^{\prime}}\left|x_{1}-x_{2}\right|}\right)+\mathscr{O}\left(\Omega^{-3}\right),(1
$$

which is equal to the disconnected part $\left\langle\rho_{1}\left(x_{1}\right) \rho_{1}\left(x_{2}\right)\right\rangle_{Q}$ at large separation, but it tends to the asymptotic value faster than the later since it only couples to the states containing $\eta^{\prime}$. Then the time-correlation function of $\eta^{\prime}$ is fitted to $A+B\left(e^{-M t}+e^{-M(T-t)}\right)$ to obtain the constant $A=$ $\frac{1}{m_{q}^{2}} \frac{1}{T}\left(\frac{Q_{t}^{2}}{\Omega}-\chi_{t}-\frac{c_{4}}{2 \chi_{t} \Omega}\right)$, and from which to extract $\chi_{t}$ provided that $\left|c_{4}\right| \ll 2 \chi_{t}^{2} \Omega$. This was how we determined the topological susceptibility in two-flavor lattice QCD with fixed topology $[9,10]$.

However, it was unclear to what extent the assumption $\left|c_{4}\right| \ll 2 \chi_{t}^{2} \Omega$ was satisfied. To eliminate this constraint, we compute the 4-point correlator of $\eta^{\prime}$, as well as the 2-point correlator. Theoretically, in a fixed topology, the former behaves as [4]

$$
\lim _{\left|x_{i}-x_{j}\right| \gg 1} m_{q}^{4}\left\langle\eta^{\prime}\left(x_{1}\right) \cdots \eta^{\prime}\left(x_{4}\right)\right\rangle_{Q}=\frac{3 \chi_{t}^{2}}{\Omega^{2}}\left(1-\frac{Q^{2}}{\chi_{t} \Omega}+\frac{c_{4}}{\chi_{t}^{2} \Omega}\right)^{2}+\mathscr{O}\left(e^{-m_{\eta^{\prime}}\left|x_{i}-x_{j}\right|}\right)+\mathscr{O}\left(\Omega^{-4}\right),
$$

From (1.6) and (1.7), one can solve for $\chi_{t}$ and $c_{4}$ (or equivalently, the parameter $y$ )

$$
\begin{aligned}
\chi_{t} & =\frac{Q^{2}}{\Omega}+\Omega\left(2 k_{2}-\sqrt{k_{4} / 3}\right), \\
y & \equiv \frac{c_{4}}{2 \chi_{t}^{2} \Omega}=-\frac{\left(\sqrt{k_{4} / 3}-k_{2}\right)}{\sqrt{k_{4} / 3}-2 k_{2}}\left(1-\frac{Q^{2}}{\chi_{t} \Omega}\right),
\end{aligned}
$$


where $-k_{2}$ and $k_{4}$ are the asymptotic values of 2-point and 4-point correlators at large separation. It is interesting to note that if one neglects the $y$ term in (1.6) and (1.7), they reduce to

$$
\begin{aligned}
& \chi_{t} \simeq \frac{Q^{2}}{\Omega}+\Omega k_{2}, \\
& \chi_{t} \simeq \frac{Q^{2}}{\Omega}+\Omega \sqrt{k_{4} / 3},
\end{aligned}
$$

which provide two independent estimates of $\chi_{t}$. In other words, if $|y| \ll 1$, then (1.8), (1.10) and (1.11) all give compatible results for $\chi_{t}$. On the other hand, if (1.10) and (1.11) turn out to be quite different from each other, then $|y|$ must be substantially larger than zero, and a more reliable estimate of $\chi_{t}$ could be given by (1.11).

For the (2+1)-flavor QCD, the $\eta^{\prime}$ interpolating operator must take into account of the fact that different flavors have different quark masses, namely,

$$
m_{q} \eta^{\prime}=\frac{m_{q}}{N_{f}} \sum_{f=1}^{N_{f}} \bar{q}_{f} \gamma_{5} q_{f} \longrightarrow \eta_{T}^{\prime}=\frac{1}{N_{f}} \sum_{f=1}^{N_{f}} m_{f} \bar{q}_{f} \gamma_{5} q_{f}
$$

where $\eta_{T}^{\prime}$ is called the "topological" $\eta^{\prime}$ operator for computing topological charge density correlators.

In this paper, we use 80 pairs of low-lying eigenmodes of the overlap operator to evaluate the 2-point and 4-point correlators of $\eta_{T}^{\prime}$, and to extract their asympototic values $-k_{2}$ and $k_{4}$. Then we use (1.8)-(1.9) to obtain $\chi_{t}$ and $y$. Note that $c_{4}$ is related to the leading anomalous contribution to the $\eta^{\prime}-\eta^{\prime}$ scattering amplitude in QCD, as well as the dependence of the vacuum energy on the vacuum angle $\theta$.

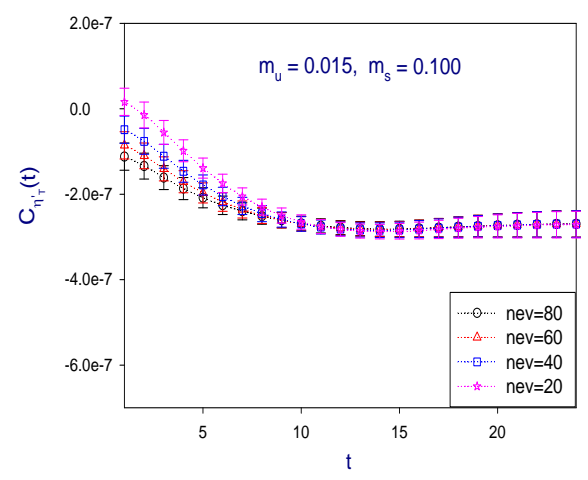

(a)

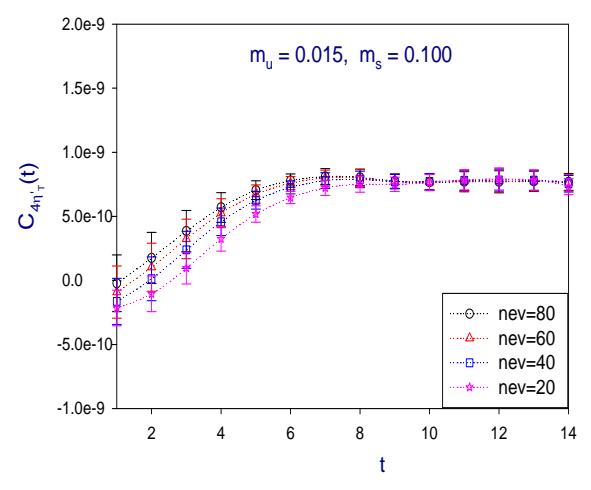

(b)

Figure 1: Low-mode saturation of (a) the 2-point function $C_{\eta_{T}^{\prime}}(t)$ (b) the 4-point function $C_{4 \eta_{T}^{\prime}}(t)$

\section{Lattice Setup}

Our simulations are carried out in the topologically-trivial sector (with $Q_{t}=0$ ) for $(2+1)$ flavor QCD on a $16^{3} \times 48$ lattice at a lattice spacing $\sim 0.11 \mathrm{fm}$ (for details, see [11] and [12]). For 
the gluon part, the Iwasaki action is used at $\beta=2.30$, together with unphysical Wilson fermions and associated twisted-mass ghosts [13]. The unphysical degrees of freedom generate a factor $\operatorname{det}\left[H_{w}^{2}\left(-m_{0}\right) /\left(H_{w}^{2}\left(-m_{0}\right)+\mu^{2}\right)\right]$ in the partition function (we take $m_{0}=1.6$ and $\mu=0.2$ ) that suppresses the near-zero eigenvalue of $H_{w}\left(-m_{0}\right)$ and thus makes the numerical operation with the overlap operator substantially faster. Furthermore, since the exact zero eigenvalue is forbidden, the global topological change is preserved during the molecular dynamics evolution of the gauge field.

For $m_{s}=0.100$, we take five sea quark mass $m_{u(d)}$ values: $0.015,0.025,0.035,0.050$, and 0.100 that cover the mass range $m_{s} / 6-m_{s}$. After discarding 500 trajectories for thermalization, we accumulate 2500 trajectories in total for each sea quark mass. In the calculation of $\chi_{t}$, we take one configuration every 5 trajectories, thus we have 500 configurations for each $m_{q}$. For each configuration, 80 pairs of lowest-lying eigenmodes of the overlap-Dirac operator $D(0)$ are calculated using the implicitly restarted Lanczos algorithm and stored for the later use.

\section{Results}

In practice, we use 80 pairs of low-lying eigenmodes of the overlap operator to evaluate the 2-point and 4-point time-correlation functions of $\eta_{T}^{\prime}$

$$
\begin{aligned}
C_{\eta_{T}^{\prime}}(t) & =\frac{1}{L^{3} T} \sum_{u=1}^{T} \sum_{\vec{x}_{i}}\left\langle\eta_{T}^{\prime}\left(\vec{x}_{2}, u+t\right) \eta_{T}^{\prime}\left(\vec{x}_{1}, u\right)\right\rangle, \quad \lim _{t \gg 1} \frac{1}{L^{3}} C_{\eta_{T}^{\prime}}(t)=-k_{2}, \\
C_{4 \eta_{T}^{\prime}}(t) & =\frac{1}{L^{3} T} \sum_{u=1}^{T} \sum_{\vec{x}_{i}}\left\langle\eta_{T}^{\prime}\left(\vec{x}_{4}, u+3 t\right) \eta_{T}^{\prime}\left(\vec{x}_{3}, u+2 t\right) \eta_{T}^{\prime}\left(\vec{x}_{2}, u+t\right) \eta_{T}^{\prime}\left(\vec{x}_{1}, u\right)\right\rangle, \quad \lim _{t \gg 1} \frac{1}{L^{9}} C_{4 \eta_{T}^{\prime}}(t)=k_{4} .
\end{aligned}
$$

Thus it is crucial to check whether these 80 eigenmodes suffice to saturate $C_{\eta_{T}^{\prime}}(t)$ and $C_{4 \eta_{T}^{\prime}}(t)$ respectively. In Fig. 1, we plot $C_{\eta_{T}^{\prime}}(t)$ and $C_{4 \eta_{T}^{\prime}}(t)$ for $m_{u}=0.015$, versus the number of eigenmodes (nev) 20,40, 60, and 80 respectively. Obviously, $C_{\eta_{T}^{\prime}}(t)$ is well saturated with 80 eigenmodes for the time range $15 \leq t \leq 24$ where it attains a plateau. Similarly, $C_{4 \eta_{T}^{\prime}}(t)$ is also well saturated for the time range $9 \leq t \leq 14$ where it attains a plateau. The low-mode saturation also holds for all five sea quark masses.

In Fig. 2, we plot the values of $\chi_{t} a^{4}(1.8)$ and $y(1.9)$ versus the sea quark mass $m_{q} a$, together with the values of $\chi_{t}$ obtained from the 2-point function (1.10) and the 4-point function (1.11) respectively. Evidently, the values of $\chi_{t}$ from (1.8), (1.10), and (1.11) are in good agreement with one another. For the smallest four quark masses, 0.015, 0.025, 0.035, and 0.050, the data points of $a^{4} \chi_{t}$ are well fitted by the ChPT formula [3]

$$
\chi_{t}=\frac{\Sigma}{m_{u}^{-1}+m_{d}^{-1}+m_{s}^{-1}},
$$

with $a^{3} \Sigma=0.0021(1)$. In order to convert $\Sigma$ to that in the $\overline{\mathrm{MS}}$ scheme, we calculate the renormalization factor $Z_{m}^{\overline{\mathrm{MS}}}(2 \mathrm{GeV})$ using the non-perturbative renormalization technique through the $\mathrm{RI} / \mathrm{MOM}$ scheme. Our result is $Z_{m}^{\overline{\mathrm{MS}}}(2 \mathrm{GeV})=0.800(10)(24)$ [14]. With $a^{-1}=1833(12) \mathrm{MeV}$ determined with $r_{0}=0.49 \mathrm{fm}$ [12], the value of $\Sigma$ is transcribed to

$$
\Sigma^{\overline{\mathrm{MS}}}(2 \mathrm{GeV})=[253(4)(6) \mathrm{MeV}]^{3}
$$




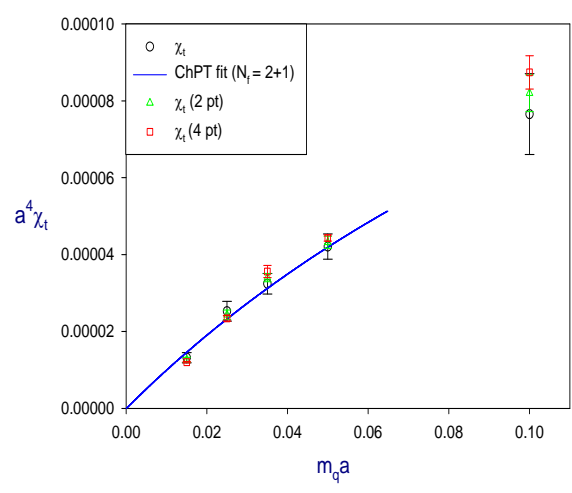

(a)

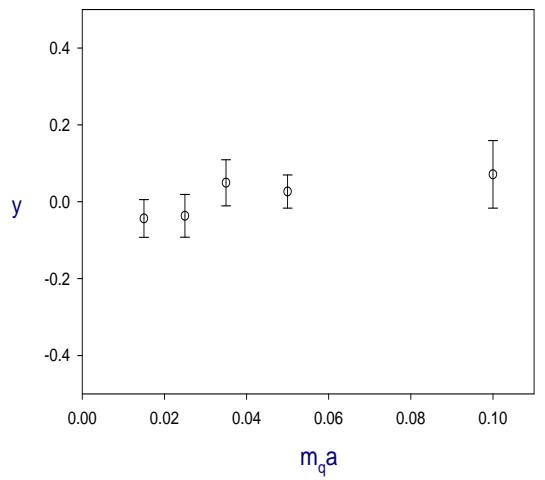

(b)

Figure 2: Topological susceptibility $\chi_{t} a^{4}$ and $y \equiv c_{4} /\left(2 \chi_{t}^{2} \Omega\right)$ versus sea quark mass $m_{q} a$ for (2+1)-flavor lattice QCD with fixed topological charge $Q_{t}=0$.

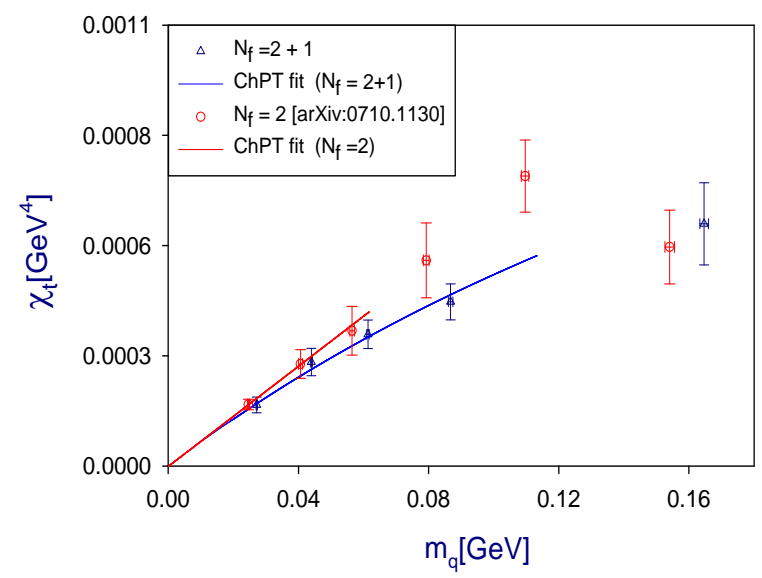

Figure 3: The topological susceptibility $\chi_{t}$ versus $m_{q}$ for lattice QCD with fixed topology $Q_{t}=0$.

which is in good agreement that extracted from $\chi_{t}=\left\langle Q_{t}^{2}\right\rangle / \Omega$ with $Q_{t}$ determined by the spectral flow method for the $2+1$ flavors QCD configurations generated by the RBC and UKQCD Collaborations with domain-wall fermions [15]. Also, it is in good agreement with our previous results extracted from $\chi_{t}$ in 2-flavor QCD $[9,10]$, and in the $\varepsilon$-regime from the low-lying eigenvalues [16]. The errors represent a combined statistical error $\left(a^{-1}\right.$ and $\left.Z_{m}^{\overline{\mathrm{MS}}}\right)$ and the systematic error respectively.

At this point, it is instructive to plot $\chi_{t}$ versus $m_{q}$, for 2-flavor QCD (data from $[9,10]$ ), and $(2+1)$-flavor QCD (this work), as shown in Fig. 3. Now we can see clearly how the topological susceptibility changes with respect to the number of flavors.

\section{Concluding remark}

In this paper, we have obtained the topological susceptibility $\chi_{t}$ and $c_{4}$ in $(2+1)$-flavor QCD from a lattice calculation of 2-point and 4-point correlators at a fixed global topological charge 
$Q_{t}=0$. The expected sea quark mass dependence of $\chi_{t}$ from ChPT is clearly observed. Our result asserts that the topologically non-trivial excitations are in fact locally active in the QCD vacuum, even when the global topological charge is zero. The information of these topological excitations is carried by the low-lying eigenmodes of the overlap Dirac operator. We will use the values of $\chi_{t}$ we have determined to remove the artifacts due to the fixed topology in a finite volume and to obtain the physical results in the $\theta$ vacuum $[17,4]$.

Finally we note that our result of the ratio $\left|c_{4}\right| / \chi_{t}$ is substantially less than one in the chiral limit, similar to its counterpart in quenched QCD $[18,19]$. This seems to suggest that the quantum corrections would suppress the emergence of dilute instanton gas in the full QCD vacuum.

Numerical simulations are performed on Hitachi SR11000 and IBM System Blue Gene Solution at High Energy Accelerator Research Organization (KEK) under a support of its Large Scale Simulation Program (No. 08-05), and also on IBM and HP clusters at NCHC and NTU-CC in Taiwan. This work is supported in part by the Grant-in-Aid of the Japanese Ministry of Education (Nos. 18340075, 18740167, 19540286, 19740160, 20025010, 20039005, 20340047, 20740156), the National Science Council of Taiwan (Nos. NSC96-2112-M-002-020-MY3, NSC96-2112-M001-017-MY3, NSC97-2119-M-002-001), and NTU-CQSE (Nos. 97R0066-65, 97R0066-69).

\section{References}

[1] E. Witten, Nucl. Phys. B 156, 269 (1979).

[2] G. Veneziano, Nucl. Phys. B 159, 213 (1979).

[3] H. Leutwyler and A. Smilga, Phys. Rev. D 46, 5607 (1992).

[4] S. Aoki, H. Fukaya, S. Hashimoto and T. Onogi, Phys. Rev. D 76, 054508 (2007)

[5] M. F. Atiyah and I. M. Singer, Annals Math. 87, 484 (1968).

[6] H. Neuberger, Phys. Lett. B 417, 141 (1998); Phys. Lett. B 427, 353 (1998)

[7] R. Narayanan and H. Neuberger, Nucl. Phys. B 443, 305 (1995)

[8] T. W. Chiu and S. V. Zenkin, Phys. Rev. D 59, 074501 (1999)

[9] T. W. Chiu et al. [JLQCD and TWQCD Collaborations], PoS LAT2007, 068 (2007)

[10] S. Aoki et al. [JLQCD and TWQCD Collaborations], Phys. Lett. B 665, 294 (2008)

[11] S. Hashimoto et al. [JLQCD collaboration], PoS LAT2007, 101 (2007)

[12] H. Matsufuru et al. [JLQCD and TWQCD Collaborations], PoS LAT2008, 077 (2008)

[13] H. Fukaya, S. Hashimoto, K. I. Ishikawa, T. Kaneko, H. Matsufuru, T. Onogi and N. Yamada [JLQCD Collaboration], Phys. Rev. D 74, 094505 (2006)

[14] J. Noaki et al. [JLQCD and TWQCD Collaborations], arXiv:0810.1360 [hep-lat].

[15] T. W. Chiu, T. H. Hsieh and P. K. Tseng [TWQCD Collaboration], Phys. Lett. B 671, 135 (2009)

[16] H. Fukaya et al. [JLQCD and TWQCD Collaborations], Phys. Rev. D 76, 054503 (2007); Phys. Rev. Lett. 98, 172001 (2007)

[17] R. Brower, S. Chandrasekharan, J. W. Negele and U. J. Wiese, Phys. Lett. B 560, 64 (2003)

[18] L. Giusti, S. Petrarca and B. Taglienti, Phys. Rev. D 76, 094510 (2007)

[19] L. Del Debbio, H. Panagopoulos and E. Vicari, J. Phys. Conf. Ser. 110, 122005 (2008) 